\title{
Correction to: A systematic review of nano formulation of natural products for the treatment of inflammatory bowel disease: drug delivery and pharmacological targets
}

\author{
Yasamin Davatgaran Taghipour ${ }^{1,2,3} \cdot$ Roodabeh Bahramsoltani $^{2,4} \cdot$ André M. Marques $^{5} \cdot$ Rozita Naseri $^{6} \cdot$ Roja Rahimi $^{4}$. \\ Pouya Haratipour ${ }^{7,8} \cdot$ Amin Iranpanah $^{9} \cdot$ Mohammad Hosein Farzaei $^{10,11} \cdot$ Mohammad Abdollahi $^{12,13}$
}

Published online: 26 November 2018

(C) Springer Nature Switzerland AG 2018

\section{Correction to: DARU J Pharm Sci}

https://doi.org/10.1007/s40199-018-0222-4

The corresponding author, Mohammad Hosein Farzaei, affiliation is Pharmaceutical Sciences Research Center, Kermanshah University of Medical Sciences, Kermanshah, Iran. The correct spelling of the 7 th author surname is Iranpanah. The full reference for 18, 33, 59, 64 and 66 is as follows:

18. Sun Q, Luan L, Arif M, Li J, Dong Q-J, Gao Y, et al. Redox-sensitive nanoparticles based on 4-aminothiophenolcarboxymethyl inulin conjugate for budesonide delivery in inflammatory bowel diseases. Carbohydrate Polymers. 2018;189:352-359.

33. Zangabad PS, Mirkiani S, Shahsavari S, Masoudi B, Masroor M, Hamed H, et al. Stimulus-responsive liposomes as smart nanoplatforms for drug delivery applications. Nanotechnology Reviews. 2018;7(1):95-122.

59. Tamura A, Ozawa K, Ohya T, Tsuyama N, Eyring EM, Masujima T. Nanokinetics of drug molecule transport into a single cell. Nanomedicine (Lond). 2006,1(3):345-350.

64. Koopaei NN, Abdollahi M. Opportunities and obstacles to the development of nanopharmaceuticals for human use. DARU Journal of Pharmaceutical Sciences; 2016,24(1): 23.

66. Mostafalou S, Mohammadi H, Ramazani A, Abdollahi M. Different biokinetics of nanomedicines linking to their toxicity; an overview. DARU Journal of Pharmaceutical Sciences; 2013.21(1): 14.

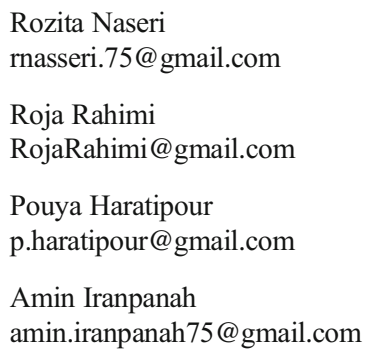

Extended author information available on the last page of the article 


\section{Affiliations}

\section{Yasamin Davatgaran Taghipour ${ }^{1,2,3} \cdot$ Roodabeh Bahramsoltani $^{2,4} \cdot$ André M. Marques $^{5} \cdot$ Rozita Naseri $^{6} \cdot$ Roja $^{\text {Rahimi }}{ }^{4}$. Pouya Haratipour ${ }^{7,8}$ - Amin Iranpanah ${ }^{9}$ - Mohammad Hosein Farzaei ${ }^{10,11}$ • Mohammad Abdollahi $^{12,13}$}

1 Department of Medical Nanotechnology, School of Advanced Medical Sciences, Tabriz University of Medical Sciences,

Tabriz, Iran

2 PhytoPharmacology Interest Group (PPIG), Universal Scientific Education and Research Network (USERN), Tehran, Iran

3 Student Research Committee, Tabriz University of Medical Sciences, Tabriz, Iran

4 Department of Pharmacy in Persian Medicine, School of Persian Medicine, Tehran University of Medical Sciences, Tehran, Iran

5 Oswaldo Cruz Foundation (FIOCRUZ), Institute of Technology in Pharmaceuticals (Farmanguinhos), Rio de Janeiro, RJ, Brazil

6 Internal Medicine Department, Faculty of Medicine, Kermanshah University of Medical Sciences, Kermanshah, Iran

7 Department of Chemistry, Sharif University of Technology, Tehran, Iran
8 PhytoPharmacology Interest Group (PPIG), Universal Scientific Education and Research Network (USERN), Los Angeles, CA, USA

9 Faculty of Pharmacy, Kermanshah University of Medical Sciences, Kermanshah, Iran

10 Pharmaceutical Sciences Research Center, Kermanshah University of Medical Sciences, Kermanshah, Iran

11 Medical Biology Research Center, Kermanshah University of Medical Sciences, Kermanshah, Iran

12 Toxicology and Diseases Group, The Institute of Pharmaceutical Sciences (TIPS), Tehran University of Medical Sciences, Tehran, Iran

13 Department of Toxicology and Pharmacology, Faculty of Pharmacy, Tehran University of Medical Sciences, Tehran, Iran 